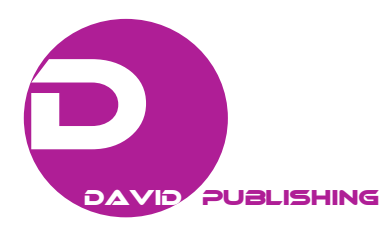

\title{
Prediction of Point in Time with High Crash Risk by Integration of Bayesian Estimation of Drowsiness, Tracking Error, and Subjective Drowsiness
}

\author{
Atsuo Murata and Yohei Uragami \\ Department of Intelligent Mechanical Systems, Graduate School of Natural Science and Technology, Okayama University, Okayama, \\ 700-8530, Japan
}

\begin{abstract}
The aim of this study was to predict drivers' drowsy states with high risk of encountering a crash and prevent drivers from continuing to drive under such drowsy states with high risk of crash. While the participants were required to carry out a simulated driving task, EEG (Electroencephalography) (EEG-MPF and EEG- $\alpha / \beta)$, ECG (Electrocradiogram) (RRV3), tracking error, and subjective rating on drowsiness were measured. On the basis of such measurements, an attempt was made to predict the point in time with high crash risk using Bayesian estimation of posterior probability of drowsiness, tracking error, and subjective drowsiness. As a result of applying the proposed method to the data of each participant, it was verified that the proposed method could predict the point in time with high crash risk before the point in time of crash.
\end{abstract}

Key words: Bayesian estimation, drowsy driving, simulated driving task, tracking error, physiological measure, crash risk.

\section{Introduction}

More and more attention has been paid to the importance of monitoring drowsiness during driving. The development of the system that can monitor drivers' arousal level and warn drivers of a risk of falling asleep is essential for the assurance of safety during driving. Advanced vehicle control systems such as longitudinal and lateral collision avoidance systems or lane keeping systems have been developed to enhance safety. Although these systems are effective to some extent, effective methods for predicting drowsiness and warning drivers of the risk of crash due caused by drowsy driving have not been established.

According to statistics by Japanese National Police Agency, the percentage of fatal crashes due to drowsy driving during 2015 is the highest and equal to $17.9 \%$ of all fatal crashes [1]. AAA (American Automobile Association) Foundation for Traffic Safety reported

Corresponding author: Atsuo Murata, professor; research fields: automotive ergonomics, transportation safety and preventive safety. E-mail: murata@iims.sys.okayama-u.ac.jp. that drowsy driving played a role in an average of 328,000 crashes annually. This total included 109,000 crashes that resulted in injuries and 6,400 fatal crashes [2]. According to FARS (Fatality Analysis Reporting System) of the US department of transportation, 416,000 crashes (fatal, injury, and PDO (property damage only)) occurred during five years from 2005 to 2009 [3]. Advanced vehicle control systems will further contribute to the enhanced traffic safety if this is used together with drowsiness prediction and warning systems.

Brown et al. [4] classified the methods for assessing drowsiness into driver-based and vehicle-based approaches. Driver-based approaches include EEG, HRV (heart rate variability), and ocular measures such as a pupil diameter and the number of blinks [5-14]. Lane position, line crossing, and steering wheel inputs are included in vehicle-based approaches.

Dinges et al. [15] and Hanowski et al. [16] showed that PERCLOS (percentage eye closure) is more reliable across drivers than EEG and eye blinks are. Ji 
et al. [17] attempted to assess a participant's drowsiness level by integrating the measures of eyelid movements, head movements, eye gazes, and facial expressions. Ji et al. [18] proposed a model of drowsiness generation and attempted to predict drowsiness. However, it was difficult to identify the point in time at which the likelihood of falling asleep increases, although the algorithms could differentiate between the drowsy and aroused states. Kusuma and Sunitha [19] incorporated a four-step system (face detection, eye detection, eye state analysis, and drowsiness detection) to detect drowsiness.

Hanowski et al. [16] showed that a multi-metric assessment system of drowsiness using an eye-closure measure and lane deviation performance is more robust and effective than a single-metric assessment system. Murata et al. [20], Murata et al. [21], and Murata et al. [22] used multiple behavioral measures such as the tracking error in a simulated driving task, back and foot pressure, and COP (center of pressure) during sitting pressure measurements, and demonstrated that multiple behavioral measures are more effective than a single behavioral or physiological measure.

Assessment of reduced arousal level or discrimination between arousal and drowsy states has been conducted by many studies. Sayed and Eskandarian [23] succeeded in achieving an accuracy of about $90 \%$ in classifying drivers as being sleep deprived or non-sleep deprived using driver steering data. Samiee et al. [24] evaluated the arousal level classified as being alert or drowsy using vehicle dynamic data such as vehicle longitudinal position and duration of eye closures, and showed that the proposed method could differentiate between the alert and the drowsy states with an accuracy of more than $87.78 \%$. Eskandarian et al. [25] and McDonald et al. [26] indicated the effectiveness of such vehicle-based measures for assessing drowsiness.

A few of the past studies on the evaluation, classification, or prediction of drowsy states have attempted to predict the point in time with high likelihood of falling asleep; however, as pointed out by Sayed et al. [27], no definite and effective methods that can determine when the alarm should be presented to the driver exist. It is possible to predict the psychological rating on drowsiness using behavioral and physiological measures [24, 28, 29]. However, currently it is impossible to predict the point in time with high crash risk (crash in simulated driving) before such a crash occurs.

Murata et al. [30] attempted to predict the risky state caused by drowsy driving using a posterior probability of drowsiness calculated on the basis of Bayesian estimation algorithm. Although the gross relationship between the posterior probability $P\left(H_{1} \mid x_{j}\right)\left(H_{1}\right.$ : drowsy, $x_{j}$ : measured data such as $E E G-M P F, E E G-\alpha / \beta$ (ration of the $\alpha$-wave power to the $\beta$-wave power), or $R R V 3$ ) and the subjective rating on drowsiness was identified, this study could not predict the point in time with high crash risk before an actual crash occurred in the simulated driving task. An increased accuracy in the prediction of the point in time with high crash risk and the automatic warning of drivers of a risky state is necessary to prevent drivers from driving under a drowsy state that might cause a crash.

This study attempted to predict the point in time with high crash risk in a driving simulator task before a virtual crash actually occurred. EEG and ECG during a simulated driving task were measured under the low arousal (drowsy) state while performing a simulated driving task. A method was proposed to predict the point in time with high risk of crash before the point in time of virtual crash occurs by means of the integration of posterior probability of drowsiness obtained by applying Bayesian estimation theorem [30], the subjective rating on drowsiness, and the tracking error. The validity of the proposed method was verified by examining whether the proposed method can predict the point in time with high crash risk before the point in time of virtual crash. 


\section{Method}

\subsection{Participants}

Thirteen male graduates or undergraduates (from 21 to 26 years old) participated in the experiment. They were all healthy and had no orthopedic or neurological diseases. All had held a driver's license for 3-4 years, and were required to stay up all night and visit our laboratory early in the morning (at about five). They were not permitted to take drink containing caffeine and exercise excessively during the sleep deprivation. The screen time such as spending in interacting with a PC or a smart phone was also confined to less than one hour during the sleep deprivation. In such a way, one can induce a condition so that the participants readily felt asleep or carry out an experimental task under a drowsy or low arousal state. The visual acuity of the participants was more than 20/20. All signed the informed consent after receiving a brief explanation on the aim and the contents of the experiment.

\subsection{Apparatus}

The apparatus, task, design and procedure were the same with Murata et al. [20-22]. EEG activities were acquired using PowerLab 8/30 (AD Instrument) and bio-amplifier ML132 (AD Instrument). Surface EEG was recorded using silver/silver chloride surface electrodes, and sampled with a sampling frequency of 1 $\mathrm{kHz}$. According to international 10-20 standard, EEGs were led from $\mathrm{O} 1$ and $\mathrm{O} 2$. ECG was led from V5 using Biolabo DL-2000 (S\&ME). The outline of display of driving simulator, steering wheel, and switch for evaluating subjective drowsiness is depicted in Fig. 1.

\subsection{Task, Design and Procedure}

The participants sat on an automobile seat, and were required to carry out a simulated driving task using a steering wheel shown in Fig. 1. The inside lane in Fig. 1 is displayed on the screen $3.2 \mathrm{~m}$ in front of the participant via a projector (EPSON, EB-S12H). They were required to steer a steering wheel and keep their own vehicle to the center line in Fig. 1 as much as they could. The driving simulator consisted of three inside lanes in one direction, and the participants were required to run the second lane. The width of each lane inside the simulated driving task was assumed to be $3.6 \mathrm{~m}$. In the simulated driving task, the tracking error between the center line in Fig. 1 and the present vehicle location (arrow in Fig. 1) was recorded every 1 s. The mean tracking error every 1 min was calculated. This was used to identify the point in time of virtual crash described in Section 3.1.

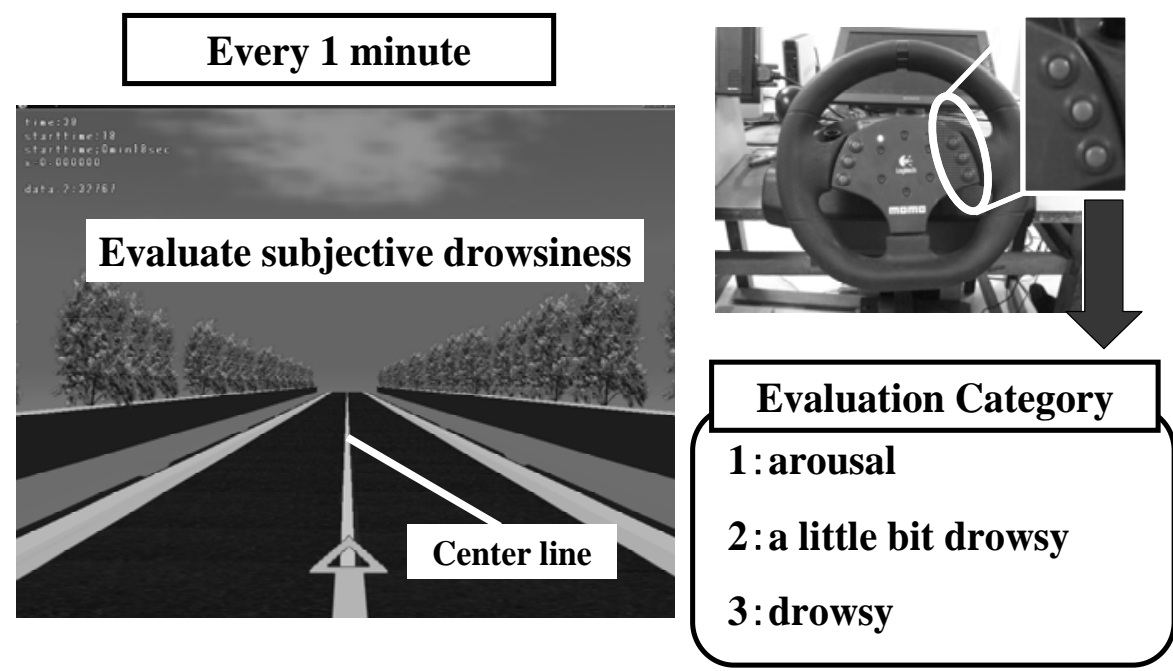

Fig. 1 Outline of display of driving simulator, steering wheel, and switch for evaluating subjective drowsiness. 
The participants were also asked to evaluate their arousal level every 1 min according to the following category: 1: arousal; 2: a little bit drowsy; 3: drowsy. It is possible to use a more detailed category such as 7-point (such as SSS (Stanford Sleepiness Scale [31]) or 9-point (such as KSS (Karolinska Sleepiness Scale [32]) category. The following advantages and disadvantages exist in such categorization. While the finer categorization enables us to conduct finer evaluation of drowsiness, the disadvantage of such categorization is that the exact evaluation according to finer categories is difficult and suffers from more frequent false (ambiguous) evaluation at the boundary of arbitrary two categories. Moreover, it is pointed out that more choice alternatives are, the less effective the choice is [33]. In this study, the participant must report their subjective rating on drowsiness every $1 \mathrm{~min}$. The more alternatives force the participants to feel it difficult to evaluate their feeling, and it is possible that the subjective evaluation cannot be carried out properly. The subjective rating on drowsiness was not a principal means for identifying the point in time with high risk of potential crash. Therefore, we adopted 3-point categorization of drowsiness. The psychological evaluation of drowsiness was incorporated into the experimental procedure to help the identification of point in time of highly risky state and virtual crash.

EEG $(E E G-M P F, E E G-\alpha / \beta)$ and heart rate variability ( $R R V 3)$ while performing a simulated driving task were derived to evaluate drowsiness. The relation between these measurements and drowsiness was analyzed. As well as the physiological measures above, the tracking error during a simulated driving task was recorded. The psychological rating of drowsiness reported every 1 min was used to grasp the change of drowsiness over time and detect a highly risky state using the tracking error, the subjective rating on drowsiness, and the result of Bayesian estimation in 2.4. The duration of each experiment ranged from $420 \mathrm{~s}$ to $2,400 \mathrm{~s}$, because the measurement was continued until the experimenter made the either of the following two judgments: (1) The participant fall asleep and is unable to carry out the experimental task any more; (2) The participant will not fall asleep if the experiment is continued further. Although the participants were required to stay up all night and the experiment began early in the morning (at about five), there were individual differences in the extent of induced drowsiness caused by staying up all night. Therefore, the duration of experimental measurement differed among participants.

\subsection{Definition of Point in Time of Virtual Crash}

A virtual crash was defined as follows. It was judged that the participant encountered a crash on the simulated driving task when the following two conditions were simultaneously satisfied: (1) Mean tracking error per minute was more than $1.8 \mathrm{~m}$; (2) The participant could not report subjective drowsiness using a switch. The check of the two conditions was visually carried out by two experimenters. The experimenters confirmed that Condition (1) was not merely due to an error in steering operation. Only when the judgment concerning the satisfaction of (1) and (2) above were consistent among the two experimenters, this was regarded as a virtual crash. Before carrying out the experiment, the two experimenters were trained so that the inter-rater (experimenter) reliability of decision making was ensured. As shown in Fig. 2, the tracking error of $1.8 \mathrm{~m}$ corresponds to the half of lane width and shows that the vertical location is dispersive to a larger extent (This is not judged to be driving normally).

\section{Method for Predicting Point in Time with High Risk of Crash}

3.1 Calculation of the Integrated Posterior Probabilities $P\left(H_{1} \mid X\right)$ Using Bayesian Theorem [30]

FFT (Fast Fourier Transform) was carried out every 1,024 data (1.024s) for EEG. Before the EEG data were entered into an FFT program, the data were passed through a cosine taper window. On the basis of such an 


\section{Renewal of prior probability}

\section{Using mean tracking error every $1 \mathrm{~min}$, prior probability was renewed every 1.}

\begin{tabular}{|c|c|c|}
\hline Mean tracking error & $P\left(H_{1}\right)$ (drowsy) & $P\left(H_{2}\right)$ (arousal) \\
\hline $0 \mathrm{~m} \sim 0.9 \mathrm{~m}$ & 0.1 & 0.9 \\
\hline $0.9 \mathrm{~m} \sim 1.8 \mathrm{~m}$ & 0.3 & 0.7 \\
\hline $1.8 \mathrm{~m} \sim 5.4 \mathrm{~m}$ & 0.7 & 0.3 \\
\hline $5.4 \mathrm{~m} \sim$ & 0.9 & 0.1 \\
\hline
\end{tabular}

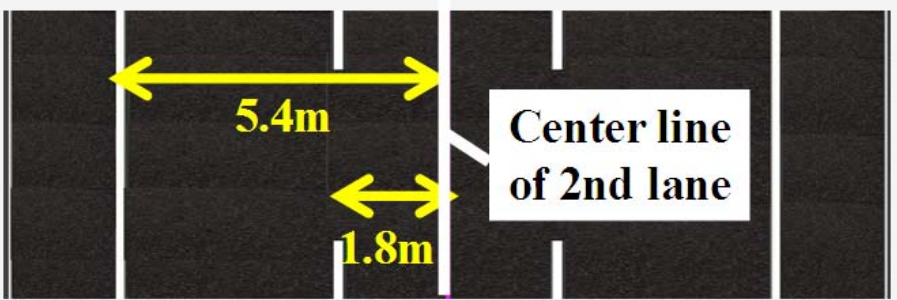

Fig. 2 Procedure for renewing prior probability according to the mean tracking error.

FFT analysis, the mean power frequency (EEG-MPF) and $E E G-\alpha / \beta$ were calculated. $E E G-M P F$ was calculated as follows:

$$
E E G-M P F=\frac{\sum_{i=1}^{30} f_{i} \cdot p o w_{i}}{\sum_{i=1}^{30} p o w_{i}}
$$

where, $f_{i}$ and pow $_{i}$ correspond to the frequency and the corresponding power, respectively. The values of $f_{1}$ and $f_{30}$ were set to about $1 \mathrm{~Hz}$ and $30 \mathrm{~Hz}$, respectively (The frequency band was assumed to range from $1 \mathrm{~Hz}$ to 30 $\mathrm{Hz}$ ). The lower value of $E E G-M P F$ shows that the arousal level is decreased. $E E G-\alpha / \beta$ can be calculated as the ration of the sum of $\alpha$-band (8-12 Hz) power and $\theta$-band (4-7 Hz) power to the power of $\beta$-band (13-30 $\mathrm{Hz}$ ) power. The higher this value is, the lower the arousal level gets.

On the basis of ECG waveform, R-R intervals (inter-beat intervals) were obtained by detecting $\mathrm{R}$ waves. HRV measure $R R V 3$ was derived as follows. The moving average per three inter-beta intervals was calculated. Variance of past three inter-beat intervals was calculated as $R R V 3$, which is regarded to represent the functions of parasympathetic nervous systems. The reason why $R R V 3$ was used to evaluate the parasympathetic system is shown in Fig. 3. The transmittance for moving average of three, five, eight, and sixteen inter-beat intervals is plotted as a function of frequency (1/beat). As shown in Fig. 3, the transmittance of $R R V 3$ for the frequency corresponding to the respiration frequency (more than 0.251 /beat) is nearly equal to zero. Therefore, $R R V 3$ can be regarded to reflect the function of parasympathetic nervous system (respiratory sinus arrhythmia). $R R V 3$ increases under the low arousal condition due to the dominance of parasympathetic nervous system.

The basic concept of Bayesian theorem [34] is depicted in Fig. 4. First, the following definitions are made before the detail of Bayesian estimation is 
described. The prior probabilities of events $H_{1}$ (drowsy) and $H_{2}$ (arousal) are given by $P\left(H_{1}\right)$ and $P\left(H_{2}\right)$. The conditional probabilities (likelihood) $P\left(x_{j} \mid H_{1}\right)$ and $P\left(x_{j} \mid H_{2}\right)$ represent the probabilities of observing the data $x_{j}$ given that the hypothesis $H_{i}$ is true. On the basis of measured data such as $x_{1}: E E G-M P F, x_{2}: E E G-\alpha / \beta$, and $x_{3}: R R V 3$, the probability of event (in this study $H_{1}$ : drowsy, $H_{2}$ : arousal) which caused the data (measurement)

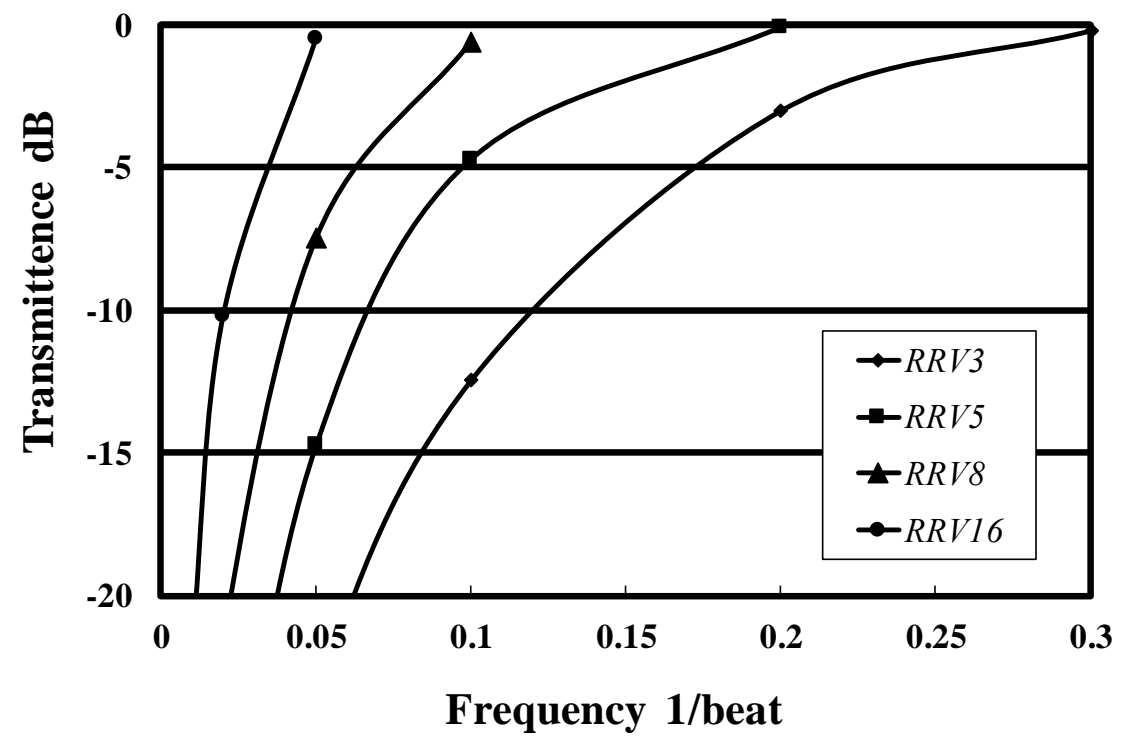

Fig. 3 Filter characteristics of RRV3 (transmittance for moving averaged time series of three, five, eight, and sixteen inter-beat intervals is plotted as a function of frequency (1/beat)).

\section{Bayesian Estimation}

On the basis of obtained data, the probability of event which caused the data given that the data is obtained is estimated using Bayesian estimation.

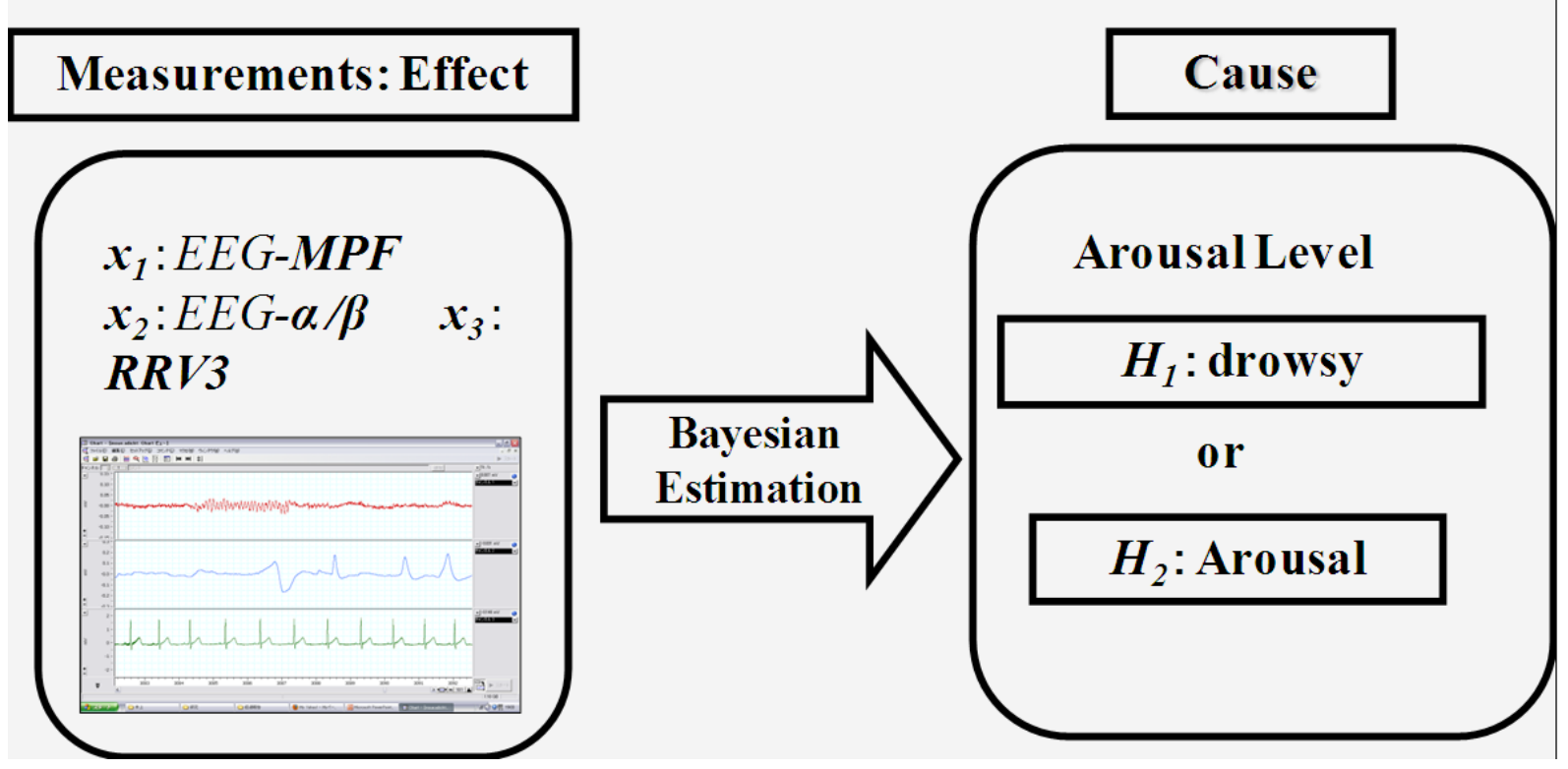

Fig. 4 Correspondence of effects (measurements $x_{1}-x_{3}$ ) and cause (events $H_{1}$ (drowsy) and $H_{2}$ (arousal)) in Bayesian estimation. 
given that the measurement is obtained (in this study, $P\left(H_{1} \mid x_{1}\right), P\left(H_{1} \mid x_{2}\right), P\left(H_{1} \mid x_{3}\right), P\left(H_{2} \mid x_{1}\right), P\left(H_{2} \mid x_{2}\right)$, and $\left.P\left(H_{2} \mid x_{3}\right)\right)$ is estimated using Bayesian estimation method below. The three measures $x_{1}, x_{2}$, and $x_{3}$ were plotted as an $X$-bar control chart (see Fig. 5). An $X$-bar control chart of $E E G-M P F$ is demonstrated in Fig. 5. Using the $X$-bar control chart [35], the judgment (assessment) of drowsiness of participants was carried out according to the procedure mentioned below.

First, the procedure for calculating the likelihood is described (see Fig. 5). In order to apply Bayesian estimation method, the likelihood $P\left(x_{j} \mid H_{i}\right)(i=1,2$; $j=1,2,3)$ was calculated as a ratio of the number of judgment (assessment) as $H_{i}$ to the total number of judgments (30 judgments) using the $X$-bar chart of each measurement. First, an interval of $30 \mathrm{~s}$ was selected. In case of $E E G-M P F$, it was assessed that the arousal level was low when more than $10 \%$ of data were below the threshold (lower control limit) $(C L-\sigma)$. In case of $E E G-\alpha / \beta$ and $R R V 3$, it was assessed that the arousal level was low when more than $10 \%$ of data were above the threshold (upper control limit) $(C L+\sigma)$. The 30s-interval was moved forward by $1 \mathrm{~s}$, and the judgment (assessment) of arousal level was carried out for the whole analysis interval. In case of EEG-based measures such as $E E G-M P F$ and $E E G-\alpha / \beta, 30 \mathrm{~s}$ and $1 \mathrm{~s}$ exactly corresponded to $30.72 \mathrm{~s}$ and $1.024 \mathrm{~s}$, respectively. As for $R R V 3,30 \mathrm{~s}$ and $1 \mathrm{~s}$ exactly corresponded to 30 beats and one beat, respectively.

Using the following Bayesian theorem, the posterior probabilities $P\left(H_{1}\right.$ (drowsy) $\left.\mid x_{j}\right)$ and $P\left(H_{2}\right.$ (arousal) $\left.\mid x_{j}\right)(j$ $=1,2,3)$ are calculated using the following formula by making use of the likelihood $P\left(x_{j} \mid H_{i}\right)(i=1,2 ; j=1,2$, 3) mentioned above (see Fig. 5). An initial value of prior probability is usually set to

$$
P\left(H_{i} \mid x_{j}\right)=P\left(H_{i}\right) P\left(x_{j} \mid H_{i}\right) / P\left(x_{j}\right)
$$

where, $P\left(H_{1}\right)=P\left(H_{2}\right)=1 / 2$, and $P\left(x_{j}\right)$ is the probability of observing $x_{j}$. According to the procedure shown in Fig. 6, the calculation of $P\left(H_{i} \mid X\right)$ (estimation of integrated posterior probability of drowsiness) according to Hershman [36] was carried out. The integration formula is given by Eq. (2). As the three measures $E E G-M P F, E E G-\alpha / \beta$, and $R R V 3$ are not

First, an interval of $30 \mathrm{~s}$ was set. In case of $E E G-M P F$, it was judged that the arousal level was low when more than $10 \%$ of data exceeded the threshold. The interval was moved forward by $1 \mathrm{~s}$, and the judgment (assessment) of arousal level was carried out for the whole interval.

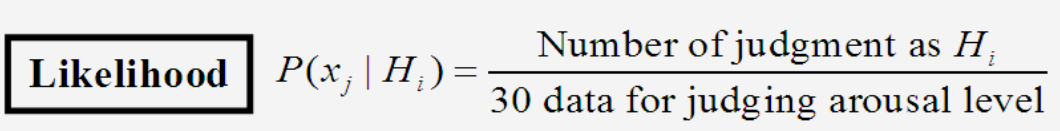

X-bar chart of $E E G-M P F$

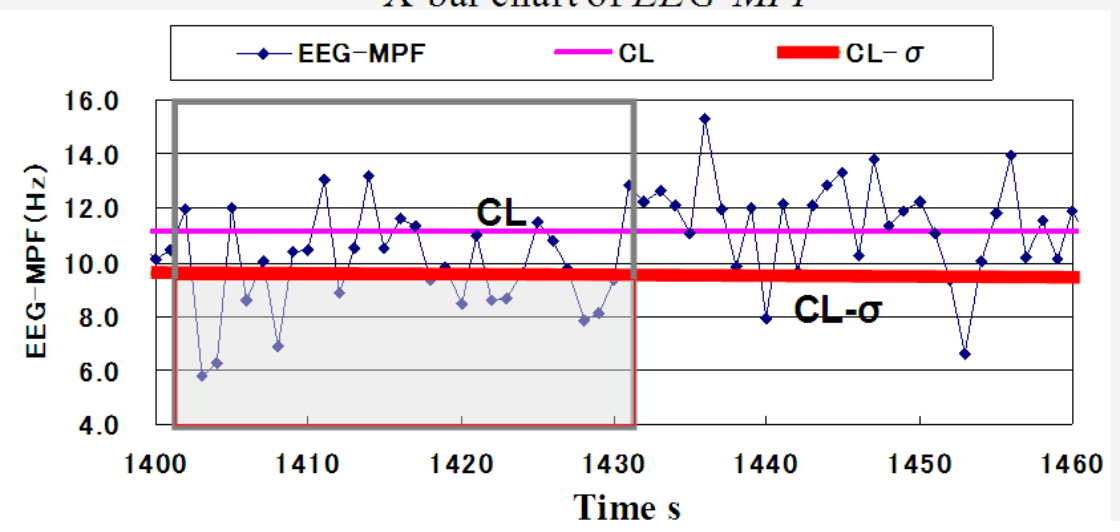

Fig. 5 Procedure for calculating likelihood necessary for Bayesian estimation using X-bar chart.

\section{Threshold \\ $L C$ : lower controllimit \\ $U C$ : upper control limit}

$x_{1}: E E G-M P F:$

$L C=C L-\sigma$

$x_{2}: E E G-\alpha / \beta$ :

$U C=C L+\sigma$

$x_{3}: R R V 3:$

$U C=C L+\sigma$

$C L$ :mean

$\sigma$ :S.D. 


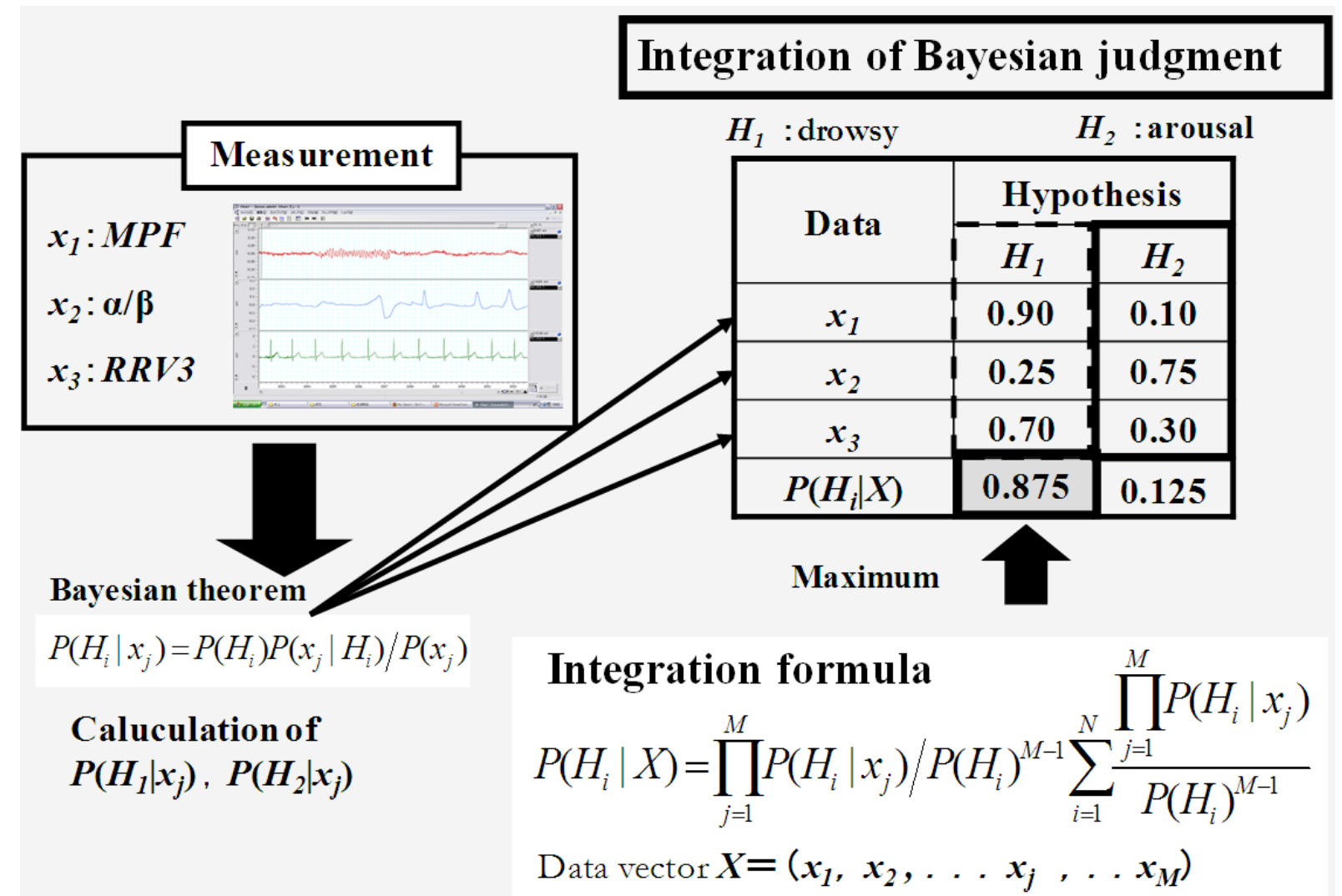

Fig. 6 Explanation for calculating $P\left(H_{1} \mid x_{j}\right)$ and $P\left(H_{2} \mid x_{j}\right)$.

highly correlated, it is possible that the integration of three measures corrects for the redundancy of a priori probability in each measure, leading to the enhanced reliability of drowsiness prediction.

$$
\begin{aligned}
P\left(H_{i} \mid x\right)= & \frac{\prod_{j=1}^{M} P\left(H_{i} \mid x_{j}\right) \prod_{j=1}^{M} P\left(x_{j}\right)}{P\left(H_{i}\right)^{M-1} \sum_{i=1}^{N} P\left(H_{i}\right) \prod_{j=1}^{M} \frac{P\left(H_{i} \mid x_{j}\right) P\left(x_{j}\right)}{P\left(H_{i}\right)}} \\
= & \frac{\prod_{j=1}^{M} P\left(H_{i} \mid x_{j}\right)}{P\left(H_{i}\right)^{M-1} \sum_{i=1}^{N} \frac{\prod_{j=1}^{M} P\left(x_{j} \mid H_{i}\right)}{P\left(H_{i}\right)^{M-1}}}
\end{aligned}
$$

where, $X=\left(x_{1}, x_{2}, \ldots, x_{M}\right)$. In this study, $M$ and $N$ are equal to 3 and 2 , respectively.

Fig. 6 demonstrates the calculated probability $P\left(H_{1} \mid x_{1}\right), P\left(H_{1} \mid x_{2}\right), P\left(H_{1} \mid x_{3}\right), P\left(H_{2} \mid x_{1}\right), P\left(H_{2} \mid x_{2}\right)$, and $P\left(H_{2} \mid x_{3}\right)$, which are used to calculate the integrated posterior probabilities $P\left(H_{1} \mid X\right)$ and $P\left(H_{2} \mid X\right)$. In the example in Fig. 6, the value of $P\left(H_{1} \mid X\right)$ is by far larger than that of $P\left(H_{2} \mid X\right)$. Here, $H_{1}$ is estimated as true. These values were calculated every second (EEG-MPF and $E E G-\alpha / \beta$ ) or inter-beat interval (RRV3).

Bayesian estimation remains unbiased with sample size due to exact estimation. Therefore, one can judge that Bayesian estimation can benefit with a limited sample size. Moreover, the proposed method mentioned in Section 3 is applied to each participant in order to predict risky and highly drowsy state of each individual. With the elapse of experiment, the data usable for Bayesian inference are accumulated. Therefore, it can be regarded that a sample size of 13 is not so inadequate.

Although an initial values of prior probability are usually set to $P\left(H_{1}\right)=P\left(H_{2}\right)=0.5$, it is necessary to renewal the prior probabilities $P\left(H_{1}\right)$ and $P\left(H_{2}\right)$ so that the estimation accuracy is enhanced. Therefore, the prior probabilities $P\left(H_{1}\right)$ and $P\left(H_{2}\right)$ were renewed every $1 \mathrm{~s}$ or 1 inter-beat interval according to the 
procedure in Fig. 2. In this manner, the integrated posterior probabilities $P\left(H_{1} \mid X\right)$ was calculated in order to make use of this for identifying the point in time with high crash risk according to the procedure in Section 3.2.

\subsection{Procedure for Identifying the Point in Time with} High Risk of Crash

The aim of this study was to verify for each participant whether it was possible to predict the highly risky state of encountering a crucial crash before the point in time of virtual crash by means of the integrated posterior probability, the subjective rating on drowsiness, and the tracking error in a simulated driving task.

The following algorithm was used for identifying the point in time with high crash risk. When Conditions (1)-(3) below were simultaneously satisfied, one can judge that it is highly risky to make the participant continue carrying out the simulated driving task:

(1) The point in time when the probability $P\left(H_{1} \mid X\right)$ is above $x(=0.8)$ occupied more than $y \%(=60 \%)$ of $z$-s interval (= $30 \mathrm{~s})$;

(2) The point in time when the mean tracking error is more than $1.8 \mathrm{~m}$ occupied more than $v \%$ (= 20\%) of $w$-s interval (= $60 \mathrm{~s})$;

(3) The rating of drowsiness corresponded to 3 (very drowsy) or the missing of response switch pressing. In addition to this, the ratings of drowsiness one minute before and after this point in time also correspond to 3 (very drowsy) or the missing of response switch pressing.

This procedure was applied to all participants, and it was checked whether the point in time with high crash risk was predictable using this procedure.

\section{Results}

\subsection{Identification of Point in Time of Virtual Crash}

As a result of checking the data of all participants according to the rule described in Section 2.4, the point in time of virtual crash was detected in eight out of thirteen participants. As for other five participants, no definite virtual crash could be identified (see Table 1). Using the proposed method, it was explored whether the proposed method can predict the point in time with high crash risk before a virtual crash actually occurred.

\subsection{Identification of Point in Time with High Crash} Risk

Examples of the change of tracking error, $P\left(H_{1} \mid X\right)$, and subjective rating on drowsiness over time and the identification of highly risky state and virtual crash are shown in Figs. 7 (Participant E), and 8 (Participant F). The result of prediction of the point in time with high

Table 1 Result of identification of point in time of virtual crash and that with high risk of crash.

\begin{tabular}{lllll}
\hline Participant & Point in time of virtual crash $x$ & Point in time of high crash risk $y$ & $x-y$ & $\begin{array}{l}\text { Percentage of drowsiness } \\
\text { evaluation of } 3 \text { or missing }\end{array}$ \\
\hline A & No detected & No detected & - & $30 \%$ \\
B & $1,200 \mathrm{~s}$ & $1,118 \mathrm{~s}$ & $82 \mathrm{~s}$ & $57.5 \%$ \\
$\mathrm{C}$ & No detected & No detected & - & $48.1 \%$ \\
$\mathrm{D}$ & $1,620 \mathrm{~s}$ & $950 \mathrm{~s}$ & $670 \mathrm{~s}$ & $57.5 \%$ \\
$\mathrm{E}$ & $360 \mathrm{~s}$ & $217 \mathrm{~s}$ & $143 \mathrm{~s}$ & $66.9 \%$ \\
$\mathrm{~F}$ & $420 \mathrm{~s}$ & $150 \mathrm{~s}$ & $270 \mathrm{~s}$ & $71.4 \%$ \\
$\mathrm{G}$ & $420 \mathrm{~s}$ & $378 \mathrm{~s}$ & $42 \mathrm{~s}$ & $71.7 \%$ \\
$\mathrm{H}$ & No detected & No detected & - & $16.1 \%$ \\
I & $1,020 \mathrm{~s}$ & $986 \mathrm{~s}$ & $34 \mathrm{~s}$ & $57.5 \%$ \\
$\mathrm{~J}$ & No detected & No detected & - & $10 \%$ \\
K & $540 \mathrm{~s}$ & $351 \mathrm{~s}$ & $189 \mathrm{~s}$ & $66.7 \%$ \\
$\mathrm{~L}$ & No detected & No detected & - & $0 \%$ \\
$\mathrm{M}$ & $1,320 \mathrm{~s}$ & $988 \mathrm{~s}$ & $332 \mathrm{~s}$ & $68.1 \%$ \\
\hline
\end{tabular}



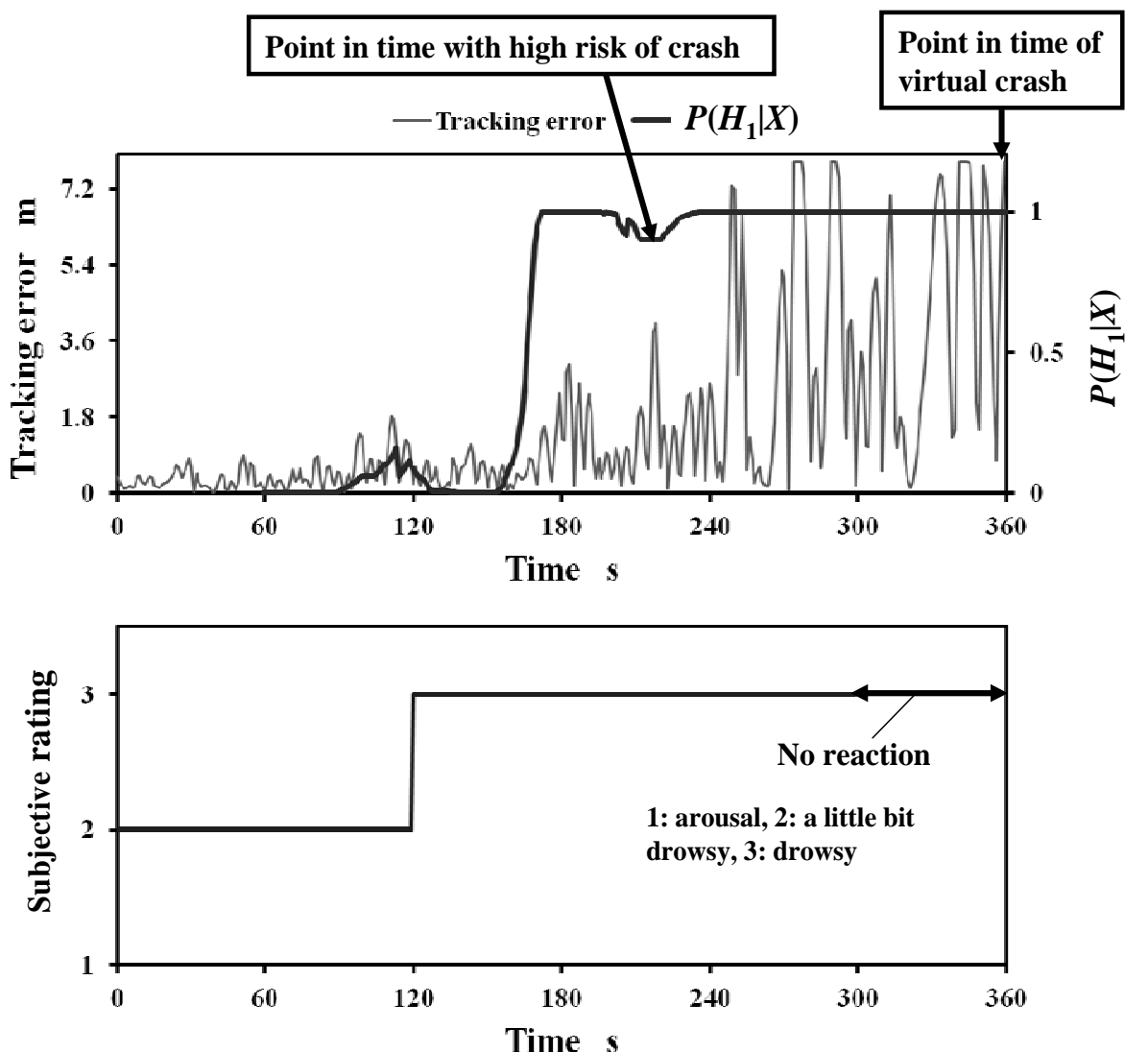

Fig. 7 Change of tracking error and $P\left(H_{1} \mid X\right)$, and subjective rating on drowsiness with time, and point in time with high risk of crash and that of virtual crash (Participant E).

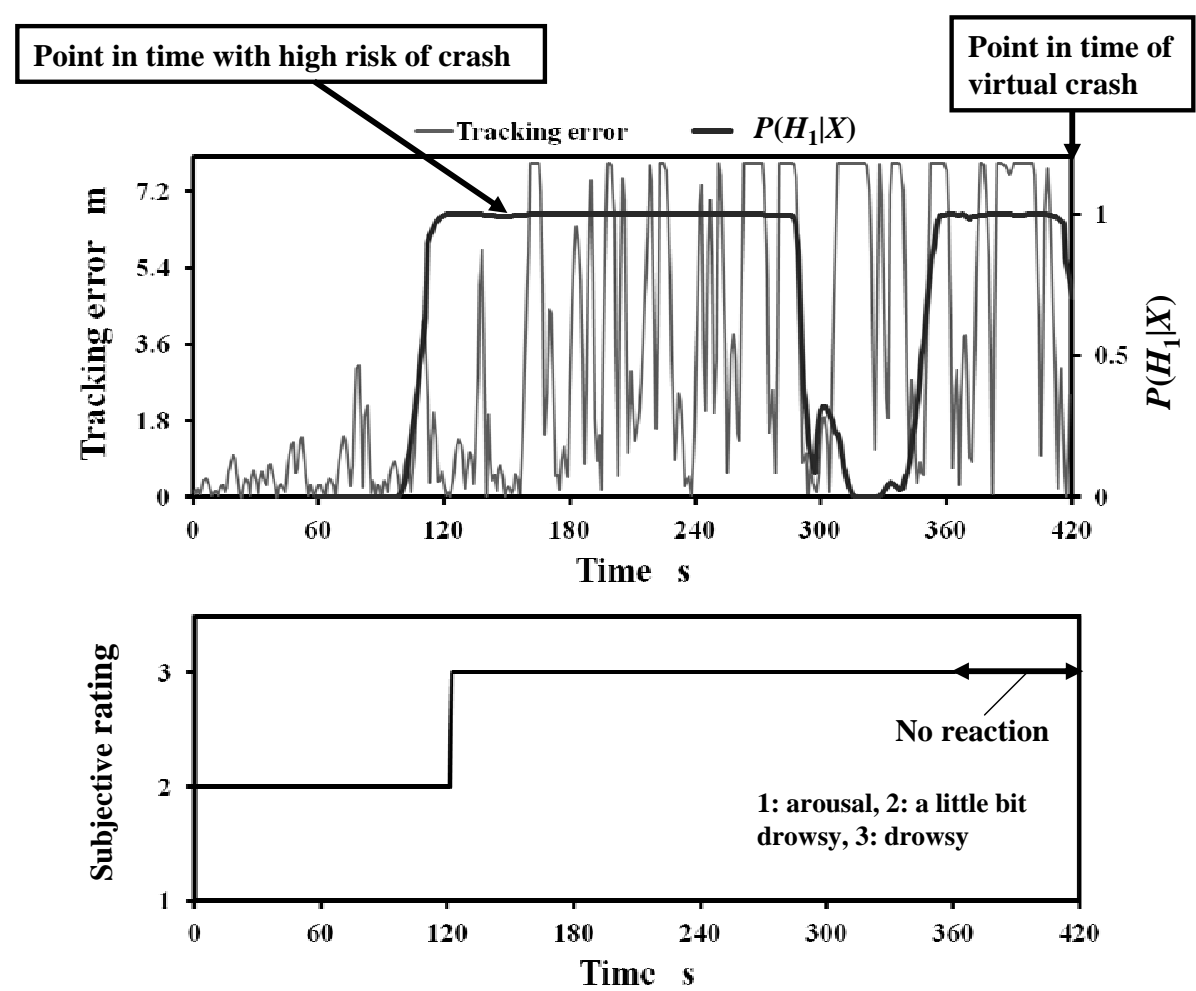

Fig. 8 Change of tracking error and $P\left(H_{1} \mid X\right)$, and subjective rating on drowsiness with time, and point in time with high risk of crash and that of virtual crash (Participant F). 
crash risk according to the procedure in Section 3.2 is summarized in Table 1.

\section{Discussion}

Although Murata et al. [29] proposed a method to calculate a posterior probability of drowsiness $P\left(H_{1} \mid X\right)$, they did not show a concrete procedure for predicting the point in time with high crash risk that might lead to a crash if the participant continues a simulated driving task. They also did not define and identify the point in time of virtual crash. This study aimed at predicting the point in time with high crash risk before a virtual crash occurs in the simulated driving task.

As shown in Table 1, the point in time with high risk of crash was detected according to the proposed procedure mentioned in Section 3.2 for all of eight participants for whom the point in time of virtual crash was detected according to the procedure mentioned in Section 2.4. On the other hand, the point in time with high crash risk was not detected for all of the five participants for whom a virtual crash did not occur. The mean percentages of drowsiness rating 3 or missing of reaction were $64.34 \%$ and $20.84 \%$ for the detected and non-detected groups of virtual crash, respectively (see
Fig. 9). The difference was statistically significant as a result of ratio test $(|t|=6.237, p<0.01)$. This means that the degree of drowsiness is larger for the participants whose point in time of virtual crash was detected than those for whom the point in time of virtual crash was not detected. Therefore, it can be judged that the procedure for predicting the point in time of high crash risk.

As for the group in which the virtual crash was detected, the time interval between point in the time of virtual crash and that of high crash risk (for eight participants to whom the virtual crash was detected) was obtained to examine whether this has some relationship with percentage of drowsiness rating 3 or missing for reaction. No definite relationship was detected as far as this study is concerned (see Fig. 10). The revelation process of drowsiness or fatigue is generally said to have a variety of patterns and be ruled by individual differences. For example, the risky state that eventually makes us fall asleep differs in its duration among individuals. To develop a more effective prediction system, a further insight into such a basic mechanism of drowsiness or fatigue would be necessary.

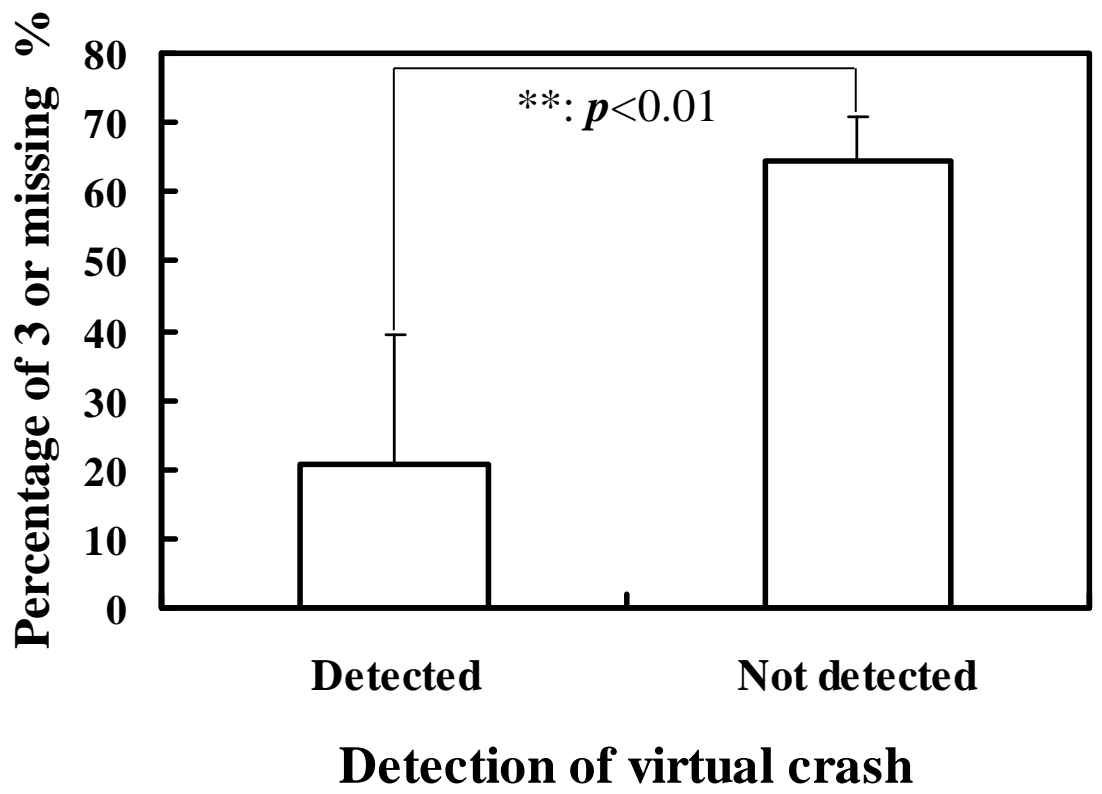

Fig. 9 Percentage of 3 or missing as a function of whether a virtual crash was detected or not. 


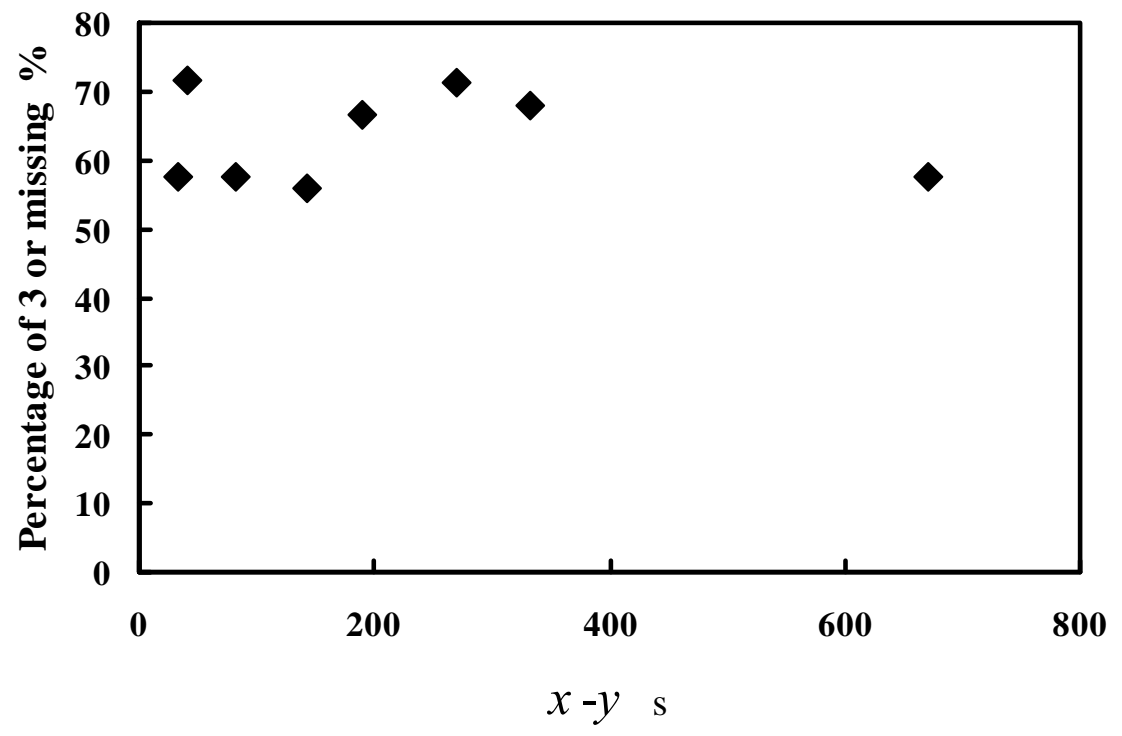

Fig. 10 Relationship between $x-y$ and percentage of 3 or missing.

Although McDonald et al. [26] showed that steering wheel angle could be used to predict drowsiness related lane-departures six seconds before they occurred, they only showed the relationship between lane-departures and drowsiness. Brookhuis and Waard [10] showed that the driver status such as drowsiness due to long-hour engagement in driving was measured by standard deviation of lateral position or steering wheel movement. From Figs.7 and 8, it is clear that the tracking error is dispersive and variable throughout the experimental duration. On the other hand, the posterior probability of drowsiness $P\left(H_{1} \mid X\right)$ was less variable and dispersive than the tracking error.

It must be noted that one cannot visually predict the point in time with high risk of crash on the basis of only lane-departure measures such as a tracking error, although this measure is to some extent effective for assessing the drowsy state. As pointed out by Hanowski et al. [16], it was judged that only a tracking error could not predict the risky state under which drivers are confronted with a higher risk of crash. This measure was used in combination with other more stable and reliable measures. Therefore, as stated in Section 3.2, an attempt was made to predict the point in time with high crash risk before a crash occurs in the simulated driving task by integrating a posterior probability $P\left(H_{1} \mid X\right)$, the tracking error, and the subjective rating on drowsiness.

As the aim of this study was to propose a method to predict the point in time with high crash risk before the point in time of virtual crash using the proposed procedure, the proposed procedure was applied to all participants so that the effectiveness of the proposed procedure can be verified. The point in time with high crash risk was not identified for the participants for whom the point in time of virtual crash was not detected. The point in time with high crash risk was identified for only participants for whom the point in time of virtual crash was detected (see Table 1). This verified the validity of the procedure for predicting the point in time with high crash risk.

The point in time with high crash risk indicates that the participant would certainly encounter a crash caused by drowsiness if such a state is left alone and no countermeasures (such as providing drivers with some alarm or warning) are taken. As shown in Table 1, the point in time with high crash risk could be detected before a virtual crash occurred. This means that the virtual crash can be prevented by predicting the point in time with high crash risk and simultaneously providing 
a participant with some alarm, or forcing to stop the simulated driving task. The prediction procedure of the point in time with high crash risk by means of the method using Bayesian estimation, the tracking error, and the subjective rating on drowsiness can be made use of so as to prevent drowsy driving.

The driving simulator corresponds to a low fidelity one. It is clear that the cognitive workload might be different between low and high fidelity simulators. The aim of this study was not to evaluate the cognitive load but to predict the point in time with high crash risk for the sleep-deprivation participants who were required to stay up all night and visit our laboratory early in the morning (at about five). Therefore, it can be judged that the fidelity of the simulator does not have a major influence on the findings.

Although the parameters $x(=0.8), y(=60 \%), z(=$ $30 \mathrm{~s}), v(=20 \%)$, and $w(=60 \mathrm{~s})$ were empirically determined in this study, future work must explore how the parameters $x, y, z, v$, and $w$ above should be systematically determined. As the participants consisted of a sample of male university students, this might limit the validity of this study. Future research should verify whether the proposed method can effectively predict the risky and drowsy state in advance for a population such as long haul truck drivers or lorry drivers.

\section{Conclusions}

The aim of this study was to predict drivers' risky state caused by drowsiness and prepare against a crash. While the participants were required to carry out a simulated driving task, EEG (MPF and $\alpha / \beta$-ratio), ECG (RRV3), tracking error, and subjective rating on drowsiness were measured. Using these measurements, a method to predict the point in time with high crash risk was proposed.

First, it was examined whether this point in time of virtual crash was observed for each participant. On the basis of such measurements, using the posterior probability of drowsiness, tracking error, and subjective rating on drowsiness, an attempt was made to predict the point in time with high crash risk before a virtual crash occurred.

The proposed procedure could effectively predict the point in time with high crash risk for only the participants for whom the point in time of virtual crash was identified. The corresponding point in time was not detected for the participants for whom the proposed procedure did not detect the point in time of virtual crash. In this manner, the validity of the proposed method was demonstrated.

Future research should further verify the effectiveness of the proposed method in more real-world environment. Using physiological measures such as $E E G-M P F, E E G-\alpha / \beta$-ratio, and RRV3 is not practical and feasible due to high price (cost) of such physiological measurement apparatus. Thus, it should be explored whether the application of the proposed method to only the behavioral drowsiness evaluation measure [20, 21, 29] can also effectively predict the point in time with high crash risk.

\section{Acknowledgments}

This work was partly supported by Grant-in-Aid for Scientific Research (B) (22310101, 26282095), Japan Society for the Promotion of Science (JSPS).

\section{References}

[1] Portal Site of Official Statistics of Japan. n.d. "Traffic Accidents Situation.” Accessed October 20, 2015. http://www.e-stat.go.jp/SG1/estat/eStatTopPortalE.do.

[2] Sleep Education for School. n.d. "Drowsy Driving Statistics.” Accessed June 20, 2016. http://school.sleepeducation.com/drowsydrivingstats.aspx

[3] NHTSA (National Highway Traffic Safety Administration). 2011. Traffic Safety Facts. Accessed July 13, 2017. http://www-nrd.nhtsa.dot.gov/Pubs/811449.pdf.

[4] Brown, D. S., Schaudt, W. A., and Hanowski, R. J. 2012. "Advances in Drowsy Driver Assistance Systems through Data Fusion.” In Handbook of Intelligent Vehicles, edited by Eskandarian, A. Vol. 2. London: Springer, 895-909.

[5] McGregorm, D. K., and Stern, J. A. 1996. "Time on Task and Blink Effects on Saccade Duration.” Ergonomics 39 
(4): 649-60.

[6] Milosevic, S. 1978. "Vigilance Performance and Amplitude of EEG Activity." Ergonomics 21 (11): 887-94.

[7] Piccoli, B., D’orso, M., Zambelli, P. L., Troiano, P., and Assint, R. 2001. “Observation Distance and Blinking Rate Measurement during On-site Investigation: New Electronic Equipment.” Ergonomics 44 (6): 668-76.

[8] Sharma, S. 2006. "Linear Temporal Characteristics of Heart Interbeat Interval as an Index of the Pilot's Perceived Risk.” Ergonomics 49 (9): 874-84.

[9] Tejero, P., and Choliz, M. 2002. "Driving on the Motorway: The Effect of Alternating Speed on Driver's Activation Level and Mental Effort.” Ergonomics 45 (9): 605-18.

[10] Brookhuis, K. A., and Waard, D. 1993. "The Use of Psychophysiology to Assess Driver Status.” Ergonomics 36 (9): 1099-110.

[11] Kecklund, G., and Akersted, T. 1993. "Sleepiness in Long Distance Truck Driving: An Ambulatory EEG Study of Night Driving.” Ergonomics 36 (9): 1007-17.

[12] Galley, N. 1993. "The Evaluation of the Electrooculogram as a Psycho-physiological Measuring Instrument in the Driver Study of Driver Behavior.” Ergonomics 36 (9): 1063-70.

[13] Wright, N., and McGown, A. 2001. "Vigilance on the Civil Flight Deck: Incidence of Sleepiness and Sleep during Long-Haul Flights and Associated Changes in Physiological Parameters.” Ergonomics 44 (1): 82-106.

[14] Skipper, J. H., and Wierwillie, W. W. 1986. "Drowsy Driver Detection Using Discrimination Analysis.” Human Factors 28 (5): 527-40.

[15] Dinges, D. F., Mallis, M. M., Maislin, G., and Powell, J. W. 1998. Final Report: Evaluation of Techniques for Ocular Measurement as an Index of Fatigue and as the Basis for Alertness Management. National Highway Traffic Safety Administration.

[16] Hanowski, R. J., Bowman, D., Alden, A., Wierwille, W. W., and Carroll, R. 2008. "PERCLOS+: Moving beyond Single-metric Drowsiness Monitors.” SAE Technical Paper 2008-01-2692.

[17] Ji, Q., Zu, Z., and Lan, P. 2004. "Real-Time Nonintrusive Monitoring and Prediction of Driver Fatigue.” IEEE Trans. on Vehicle Technology 53 (4): 1052-68.

[18] Ji, Q., Lan, P., and Looney, C. 2006. “A Probabilistic Framework for Modeling and Real-Time Monitoring Human Fatigue." IEEE Trans. on System, Man, and Cybernetics, Part A: Systems and Humans 36 (5): 862-75.

[19] Kusuma, K. B. M., and Sunitha, K. M. 2014. "Non Intrusive Drowsy Driver Detection.” International Journal of Advanced Trends in Computer Science and Engineering 3 (2): 32-5.
[20] Murata, T. K., Ohkubo, Y., and Moriwaka, M. 2013. "Verification of Physiological or Behavioral Evaluation Measures Suitable for Predicting Drivers’ Drowsiness.” In Proceedings of SICE2013, 1766-71.

[21] Murata, A., Nakatsuka, A., and Moriwaka, M. 2013. "Effectiveness of Back and Foot Pressures for Assessing Drowsiness of Drivers.” In Proceedings of SICE2013, 1754-9.

[22] Murata, A., Urakami, Y., Koriyama, T., and Ikeda, M. 2013. "Evaluation of Drowsiness of Driver Based on Change of Sitting Pressure Center." In Proceedings of SICE2013, 1760-5.

[23] Sayed, R., and Eskandarian, A. 2001. "Unobtrusive Drowsiness Detection by Neural Network Learning of Driver Steering." Proc. of the Institution of Mechanical Engineers Part D: Journal of Automobile Engineering 215 (D9): 969-75.

[24] Samiee, S., Azadi, S., Kazemi, R., Nahvi, A., and Eichberger, A. 2014. "Data Fusion to Develop a Driver Drowsiness Detection System with Robustness to Signal Loss.” Sensors 14: 17832-47.

[25] Eskandarian, A., Mortazavi, A., and Sayed, R. K. 2012. "Drowsy and Fatigue Driving Problem Significance and Detection Based on Driver Control Functions.” In Handbook of Intelligent Vehicles, edited by Eskandarian, A. Vol. 2. London: Springer, 941-74.

[26] McDonald, A. D., Schwartz, C., Lee, J. D., and Brown, T. L. 2012. "Real-Time Detection of Drowsiness Related Lane Departures Using Steering Wheel Angle." In Proceedings of the Human Factors and Ergonomic Society 56th Annual Meeting, 2201-5.

[27] Sayed, R. A., Eskandarian, A., and Mortazavi, A. 2012. "Drowsy and Fatigued Driver Warning, Counter Measures, and Assistance.” In Handbook of Intelligent Vehicles, edited by Eskandarian, A. Vol. 2. London: Springer, 977-90.

[28] Murata, A., Ohkubo, Y., Moriwaka, M., and Hayami, T. 2011. "Prediction of Drowsiness Using Multivariate Analysis of Biological Information and Driving Performance.” In Proceedings of SICE2011, 52-7.

[29] Murata, A., and Koriyama, T. 2012. "Basic Study on the Prevention of Drowsy Driving using the Change of Neck Bending Angle and the Sitting Pressure Distribution.” In Proceedings of SICE2012, 274-9.

[30] Murata, A. 2016. "Prediction of High Risk of Drowsy Driving by a Bayesian Estimation Method-An Attempt to Prevent Traffic Accidents due to Drowsy Driving.” In Ergonomics and Human Factors in Safety Management, edited by Carvalho, P., and Arezes, P. Boca Raton, USA: CRC Press, 354-71.

[31] Hoddes, E., Zarcone, V., Smythe, H., Phillips, R., and Dement, W. C. 1973. "Quantification of Sleepiness: A 
New Approach.” Psychophysiology 10: 431-6.

[32] Åkerstedt, T., and Gillberg, M. 1990. "Subjective and Objective Sleepiness in the Active Individual." International Journal of Neuroscience 52: 29-37.

[33] Schwartz, B. 2005. The Paradox of Choice: Why More Is Less. NY: New York, Harper Perennial.
[34] Swinburne, R. 2002. Bayes's Theorem. New York: Oxford University Press.

[35] Stamatis, D. H. 2002. Six Sigma and beyond_Design for Six Sigma. Boca Raton: St.Lucie Press, 273-5.

[36] Hershman, R. L. 1971. "A Rule for the Integration of Bayesian Options.” Human Factors 13 (3): 255-9. 